\title{
DAMA/LIBRA results and perspectives
}

\author{
R. Bernabei, P. Belli, S. d'Angelo, A. Di Marco, F. Montecchia* \\ Dip. di Fisica, Univ. "Tor Vergata" \\ and INFN-Roma "Tor Vergata", I-00133 Rome, Italy \\ F. Cappella, A. d'Angelo, A. Incicchitti \\ Dip. di Fisica, Univ. di Roma "La Sapienza" \\ and INFN-Roma, I-00185 Rome, Italy \\ V. Caracciolo, S. Castellano, R. Cerulli \\ Laboratori Nazionali del Gran Sasso, I.N.F.N., Assergi, Italy \\ C.J. Dai, H.L. He, X.H. Ma, X.D. Sheng, R.G. Wang, Z.P. Ye ${ }^{\dagger}$ \\ IHEP, Chinese Academy, P.O. Box 918/3, Beijing 100039, China
}

August 2, 2018

\begin{abstract}
The DAMA/LIBRA experiment is composed by about $250 \mathrm{~kg}$ of highly radiopure $\mathrm{NaI}(\mathrm{Tl})$. It is in operation at the underground Gran Sasso National Laboratory of the INFN. The main aim of the experiment is to investigate the Dark Matter (DM) particles in the Galactic halo by exploiting the model independent DM annual modulation signature. The DAMA/LIBRA experiment and the former DAMA/NaI (the first generation experiment having an exposed mass of about 100 $\mathrm{kg}$ ) have released results corresponding to a total exposure of 1.17 ton $\times$ yr over 13 annual cycles; they have provided a model independent evidence of the presence of DM particles in the galactic halo at $8.9 \sigma$ C.L.. The results of a further annual cycle, concluding the DAMA/LIBRA-phase1, have been released after this Workshop and are not included here. In the fall 2010 an important upgrade of the experiment have been performed. All the PMTs of the $\mathrm{NaI}(\mathrm{Tl})$ detectors have been replaced with new ones having higher quantum efficiency with the aim to decrease the software energy threshold considered in the data analysis. The perspectives of the running DAMA/LIBRAphase 2 will be shortly summarized.
\end{abstract}

\footnotetext{
*also Dip. di Ingegneria Civile e Ingegneria Informatica, Univ. "Tor Vergata", I-00133 Rome, Italy

$\dagger$ also University of Jing Gangshan, Jiangxi, China
} 


\section{The DAMA/LIBRA set-up}

The DAMA project develops and uses low background scintillators. It consists of the following experimental set-ups: i) DAMA/NaI $(\simeq 100 \mathrm{~kg}$ of highly radiopure $\mathrm{NaI}(\mathrm{Tl})$ ) that took data for 7 annual cycles and completed its data taking on July 2002 [1, 2, 3, 4, 5, 6, ;i) DAMA/LXe, $\simeq 6.5 \mathrm{~kg}$ liquid Kr-free Xenon enriched either in ${ }^{129} \mathrm{Xe}$ or in ${ }^{136} \mathrm{Xe}$ [7]; iii) DAMA/R\&D, a facility dedicated to tests on prototypes and to perform experiments developing and using various kinds of low background crystal scintillators in order to investigate various rare processes [8]; iv) DAMA/Ge, where sample measurements and measurements dedicated to the investigation of several rare processes are carried out as well as in the LNGS STELLA facility [9]; v) DAMA/CRYS, a new small set-up to test prototype detectors; vi) the second generation DAMA/LIBRA set-up, $\simeq 250 \mathrm{~kg}$ highly radiopure $\mathrm{NaI}(\mathrm{Tl}))$ [10, 11, 12, 13, 14, 15, 16, 17, 18. Many rare processes have been studied with these set-ups obtaining competitive results.

The main apparatus, DAMA/LIBRA, is investigating the presence of DM particles in the galactic halo by exploiting the model independent DM annual modulation signature. In fact, as a consequence of its annual revolution around the Sun, which is moving in the Galaxy traveling with respect to the Local Standard of Rest towards the star Vega near the constellation of Hercules, the Earth should be crossed by a larger flux of Dark Matter particles around $\sim 2$ June (when the Earth orbital velocity is summed to the one of the solar system with respect to the Galaxy) and by a smaller one around $\sim 2$ December (when the two velocities are subtracted). This DM annual modulation signature is very distinctive since the effect induced by DM particles must simultaneously satisfy all the following requirements: (1) the rate must contain a component modulated according to a cosine function; (2) with one year period; (3) with a phase that peaks roughly around $\sim$ 2nd June; (4) this modulation must be present only in a welldefined low energy range, where DM particles can induce signals; (5) it must be present only in those events where just a single detector, among all the available ones in the used set-up, actually "fires" (single-hit events), since the probability that DM particles experience multiple interactions is negligible; (6) the modulation amplitude in the region of maximal sensitivity has to be $\lesssim 7 \%$ in case of usually adopted halo distributions, but it may be significantly larger in case of some particular scenarios such as

e.g. those in refs. [19, 20]. At present status of technology it is the only model independent signature available in direct Dark Matter investigation that can be effectively exploited.

The DAMA/LIBRA data released at time of this Workshop correspond 
to six annual cycles for an exposure of 0.87 ton $\times$ yr [11, 12]. Considering these data together with those previously collected by DAMA/NaI over 7 annual cycles $(0.29$ ton $\times y r)$, the total exposure collected over 13 annual cycles is 1.17 ton $\times$ yr; this is orders of magnitude larger than the exposures typically collected in the field.

The DAMA/NaI set up and its performances are described in ref. 1, 3, [5, 21], while the DAMA/LIBRA set-up and its performances are described in ref. [10, 12]. The sensitive part of the DAMA/LIBRA set-up is made of 25 highly radiopure $\mathrm{NaI}(\mathrm{Tl})$ crystal scintillators placed in a 5 -rows by 5-columns matrix; each crystal is coupled to two low background photomultipliers working in coincidence at single photoelectron level. The detectors are placed inside a sealed copper box flushed with HP nitrogen and surrounded by a low background and massive shield made of $\mathrm{Cu} / \mathrm{Pb} / \mathrm{Cd}-$ foils/polyethylene/paraffin; moreover, about $1 \mathrm{~m}$ concrete (made from the Gran Sasso rock material) almost fully surrounds (mostly outside the barrack) this passive shield, acting as a further neutron moderator. The installation has a 3-fold levels sealing system which excludes the detectors from environmental air. The whole installation is air-conditioned and the temperature is continuously monitored and recorded. The detectors' responses range from 5.5 to 7.5 photoelectrons $/ \mathrm{keV}$. Energy calibrations with $\mathrm{X}$-rays $/ \gamma$ sources are regularly carried out down to few $\mathrm{keV}$ in the same conditions as the production runs. A software energy threshold of $2 \mathrm{keV}$ is considered. The experiment takes data up to the $\mathrm{MeV}$ scale and thus it is also sensitive to high energy signals. For all the details see ref. [10].

\section{Short summary of the results}

Several analyses on the model-independent DM annual modulation signature have been performed (see Refs. [11, 12, 13] and references therein). Here Fig. 1 shows the time behaviour of the experimental residual rates of the single-hit events collected by DAMA/NaI and by DAMA/LIBRA in the (2-6) keV energy interval [11, 12. The superimposed curve is the cosinusoidal function: $A \cos \omega\left(t-t_{0}\right)$ with a period $T=\frac{2 \pi}{\omega}=1 \mathrm{yr}$, with a phase $t_{0}=152.5$ day (June $2^{\text {nd }}$ ), and modulation amplitude, $A$, obtained by best fit over the 13 annual cycles. The hypothesis of absence of modulation in the data can be discarded [11, 12] and, when the period and the phase are released in the fit, values well compatible with those expected for a DM particle induced effect are obtained; for example, in the cumulative (2-6) keV energy interval: $A=(0.0116 \pm 0.0013) \mathrm{cpd} / \mathrm{kg} / \mathrm{keV}, T=(0.999 \pm 0.002) \mathrm{yr}$ and $t_{0}=(146 \pm 7)$ day. Summarizing, the analysis of the single-hit residual rate favours the presence of a modulated cosine-like behaviour with proper 


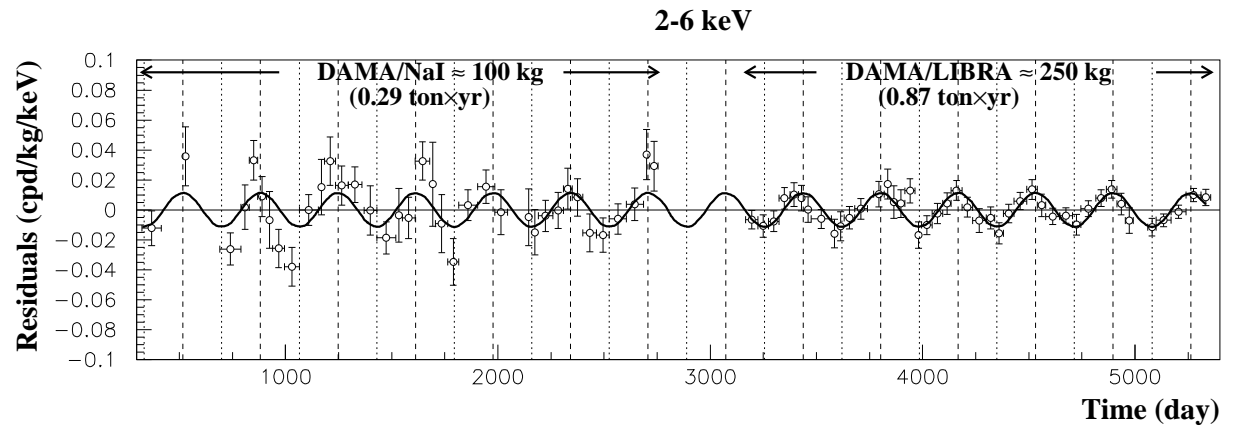

Figure 1: Experimental model-independent residual rate of the singlehit scintillation events, measured by DAMA/NaI over seven and by DAMA/LIBRA over six annual cycles in the $(2-6) \mathrm{keV}$ energy interval as a function of the time [5, 21, 11, 12. The zero of the time scale is January $1^{\text {st }}$ of the first year of data taking. The experimental points present the errors as vertical bars and the associated time bin width as horizontal bars. See refs. [11, 12 and text.

features at $8.9 \sigma$ C.L. 12 .

The same data of Fig. 1 1have also been investigated by a Fourier analysis obtaining a clear peak corresponding to a period of 1 year [12]; this analysis in other energy regions shows instead only aliasing peaks. It is worth noting that for this analysis the original formulas in Ref. [23. have been slightly modified in order to take into account for the different time binning and the residuals errors (see e.g. Ref. [13]).

Moreover, in order to verify absence of annual modulation in other energy regions and, thus, to also verify the absence of any significant background modulation, the time distribution in energy regions not of interest for DM detection has also been investigated: this allowed the exclusion of background modulation in the whole energy spectrum at a level much lower than the effect found in the lowest energy region for the single-hit events [12]. A further relevant investigation has been done by applying the same hardware and software procedures, used to acquire and to analyse the single-hit residual rate, to the multiple-hits events in which more than one detector "fires". In fact, since the probability that a DM particle interacts in more than one detector is negligible, a DM signal can be present just in the single-hit residual rate. Thus, this allows the study of the background behaviour in the same energy interval of the observed positive effect. The result of the analysis is reported in Fig. 2 where it is shown the residual rate of the single-hit events measured over the six DAMA/LIBRA annual cycles, as collected in a single annual cycle, together with the residual rates of the multiple-hits events, in the same considered energy interval. A clear 


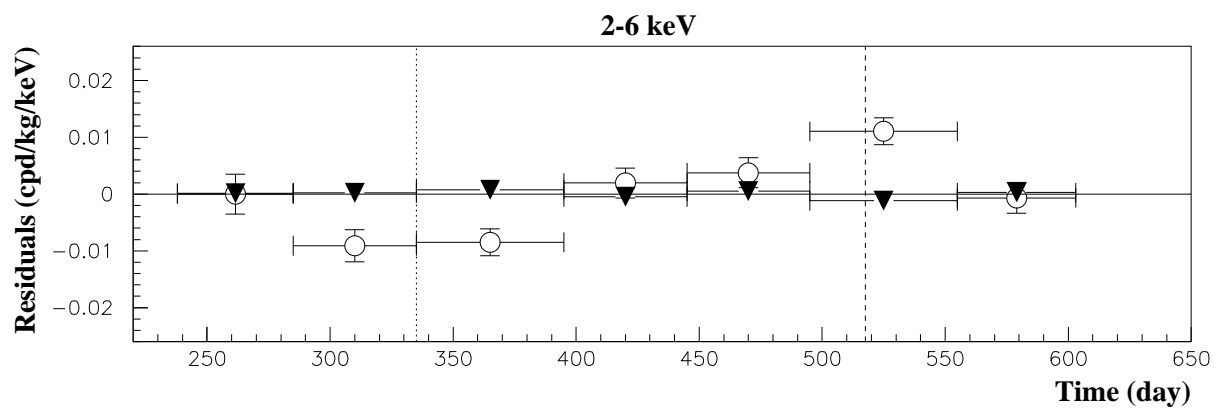

Figure 2: Experimental residual rates over the six DAMA/LIBRA annual cycles for single-hit events (open circles) (class of events to which DM events belong) and for multiple-hit events (filled triangles) (class of events to which DM events do not belong). The initial time of the figure is taken on August $7^{t h}$. The experimental points present the errors as vertical bars and the associated time bin width as horizontal bars. See text and refs. [11, 12].

modulation is present in the single-hit events, while the fitted modulation amplitudes for the multiple-hits residual rate are well compatible with zero [12. Similar results were previously obtained also for the DAMA/NaI case [21. Thus, again evidence of annual modulation with proper features, as required by the DM annual modulation signature, is present in the singlehit residuals (events class to which the DM particle induced events belong), while it is absent in the multiple-hits residual rate (event class to which only background events belong). Since the same identical hardware and the same identical software procedures have been used to analyze the two classes of events, the obtained result offers an additional strong support for the presence of a DM particle component in the galactic halo further excluding any side effect either from hardware or from software procedures or from background.

The annual modulation present at low energy has also been analyzed by depicting the differential modulation amplitudes, $S_{m}$, as a function of the energy; the $S_{m}$ is the modulation amplitude of the modulated part of the signal obtained by maximum likelihood method over the data, considering $T=1 \mathrm{yr}$ and $t_{0}=152.5$ day. The $S_{m}$ values are reported as function of the energy in Fig. 3. It can be inferred that a positive signal is present in the (2-6) keV energy interval, while $S_{m}$ values compatible with zero are present just above; in particular, the $S_{m}$ values in the (6-20) keV energy interval have random fluctuations around zero with $\chi^{2}$ equal to 27.5 for 28 degrees of freedom. It has been also verified that the measured modulation amplitudes are statistically well distributed in all the crystals, in all the 


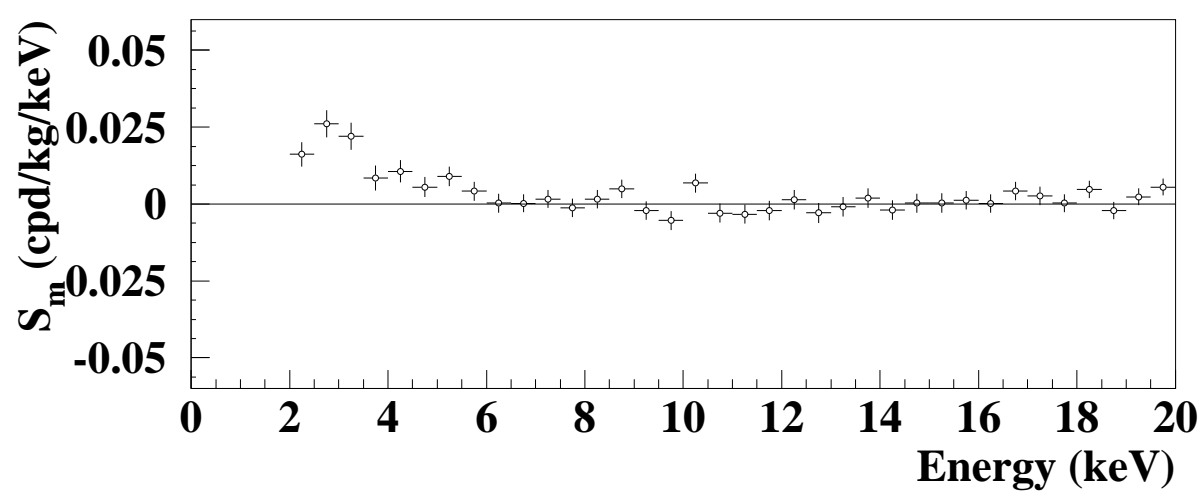

Figure 3: Energy distribution of the modulation amplitudes $S_{m}$ for the total cumulative exposure 1.17 ton $\times$ yr obtained by maximum likelihood analysis. The energy bin is $0.5 \mathrm{keV}$. A clear modulation is present in the lowest energy region, while $S_{m}$ values compatible with zero are present just above. See refs. [11, 12] and text.

annual cycles and energy bins; these and other discussions can be found in ref. [12].

Many other analyses and discussions can be found in Refs. [11, 12, 13 and references therein. Both the data of DAMA/LIBRA and of DAMA/NaI fulfil all the requirements of the DM annual modulation signature.

Careful investigations on absence of any significant systematics or side reaction have been quantitatively carried out (see e.g. Ref. [5, 3, 10, 11, 12, 17, 13, 24, 25, 26, 27, 28, 29, 30, and references therein). No systematics or side reactions able to mimic the signature (that is, able to account for the measured modulation amplitude and simultaneously satisfy all the requirements of the signature) has been found or suggested by anyone over more than a decade.

The obtained DAMA model independent evidence is compatible with a wide set of scenarios regarding the nature of the DM candidate and related astrophysical, nuclear and particle Physics. For examples some given scenarios and parameters are discussed e.g. in Ref. [2, 3, 4, 5, 11, 13. Further large literature is available on the topics (see for example in Ref [13]). Moreover, both the negative results and all the possible positive hints, achieved so-far in the field, are largely compatible with the DAMA modelindependent DM annual modulation results in many scenarios considering also the existing experimental and theoretical uncertainties; the same holds for indirect approaches; see e.g. arguments in Ref. [13] and references therein. As an example in Fig. 4 there are shown allowed regions for DM candidates interacting by elastic scattering on target-nuclei with spin- 
independent coupling, including also some of the existing uncertainties [31].

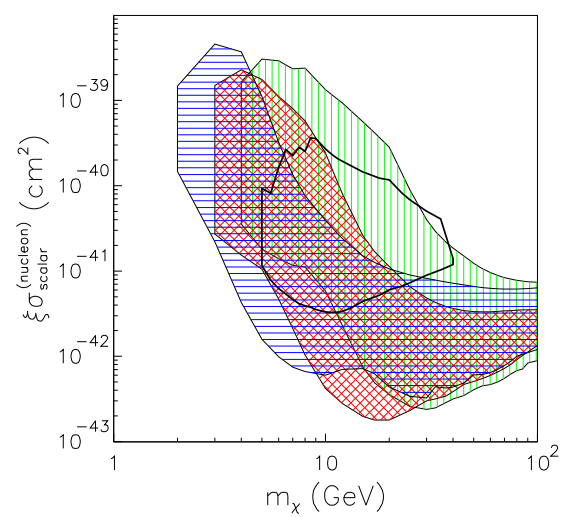

Figure 4: Regions in the nucleon cross section vs DM particle mass plane allowed by DAMA for a DM candidate interacting via spin-independent elastic scattering on target-nucleus; three different instances for the $\mathrm{Na}$ and I quenching factors have been considered: i) without including the channeling effect [(green) vertically-hatched region], ii) by including the channeling effect [(blue) horizontally-hatched region)], and iii) without the channeling effect considering energy-dependence of $\mathrm{Na}$ and I quenching factors [31] [(red) crosshatched region]. The velocity distributions and the same uncertainties as in refs. [5, 21] are considered here. These regions represent the domain where the likelihood-function values differ more than $7.5 \sigma$ from the null hypothesis (absence of modulation). The allowed region obtained for the CoGeNT experiment, including the same astrophysical models as in refs. [5, 21] and assuming for simplicity a fixed value for the Ge quenching factor and a Helm form factor with fixed parameters, is also reported by a (black) thick solid line. This region includes configurations whose likelihood-function values differ more than $1.64 \sigma$ from the null hypothesis (absence of modulation). For details see ref. [31.

\section{DAMA/LIBRA-phase2 and perspectives}

A first upgrade of the DAMA/LIBRA set-up was performed in September 2008. One detector was recovered by replacing a broken PMT and a new optimization of some PMTs and HVs was done; the transient digitizers were replaced with new ones (the U1063A Acqiris 8-bit 1GS/s DC270 HighSpeed cPCI Digitizers) having better performances and a new DAQ with optical read-out was installed. The DAMA/LIBRA-phase1 concluded its data taking in this configuration on 2010; the data of the last (seventh) 
annual cycle of this phase1 have been released after this Workshop [35.

A further and more important upgrade has been performed at the end of 2010 when all the PMTs have been replaced with new ones having higher Quantum Efficiency (Q.E.), realized with a special dedicated development by HAMAMATSU co.. Details on the developments and on the reached performances in the operative conditions are reported in Ref. [18. We remind that up to October 2010 low background PMTs, developed by EMIElectron Tubes with dedicated R\&D, were used; the light yield and other response features already allowed a software energy threshold of $2 \mathrm{keV}$ in the data analysis. The feasibility to decrease the software energy threshold below $2 \mathrm{keV}$ in the new configuration has been demonstrated [18].

Since the fulfillment of this upgrade, the DAMA/LIBRA-phase2 is continuously running in order: (1) to increase the experimental sensitivity lowering the software energy threshold of the experiment; (2) to improve the corollary investigation on the nature of the DM particle and related astrophysical, nuclear and particle physics arguments; (3) to investigate other signal features. This requires long and heavy full time dedicated work for reliable collection and analysis of very large exposures, as DAMA collaboration has always done.

Another upgrade at the end of 2012 was successfully concluded: newconcept preamplifiers were installed, with suitable operative and electronic features; in particular, they allow the direct connection of the signal to the relative channel of the Transient Digitizer (TD).

Moreover, further improvements are planned. In particular, new trigger modules have been prepared and ready to be installed. A further simplification of the electronic chain has been proposed and funded; for such purpose a new electronic module, New Linear FiFo (NLF), has been designed. It will allow - among the others - a significant reduction of the number of used NIM slots with definitive advantage.

In the future DAMA/LIBRA will also continue its study on several other rare processes [14, 15, 16] as also the former DAMA/NaI apparatus did [6].

Finally, further improvements to increase the sensitivity of the set-up are under evaluation; in particular, the use of high Q.E. and ultra-low background PMTs directly coupled to the $\mathrm{NaI}(\mathrm{Tl})$ crystals is considered 1 . This possible configuration will allow a further large improvement in the light collection and a further lowering of the software energy threshold. Moreover, efforts towards a possible highly radiopure $\mathrm{NaI}(\mathrm{Tl})$ "general purpose" experiment (DAMA/1ton) having full sensitive mass of 1 ton (we already

\footnotetext{
${ }^{1}$ However, this would require the disassembling of the detectors since the light guides act at present also as optical windows.
} 
proposed in 1996 as general purpose set-up) are continuing in various aspects.

\section{References}

[1] R. Bernabei et al., Il Nuovo Cim. A 112 (1999) 545;

[2] R. Bernabei et al., Phys. Lett. B 389 (1996) 757; Phys. Lett. B 424 (1998) 195; Phys. Lett. B 450 (1999) 448; Phys. Rev. D 61 (2000) 023512; Phys. Lett. B 480 (2000) 23; Phys. Lett. B 509 (2001) 197; Eur. Phys. J. C 23 (2002) 61; Phys. Rev. D 66 (2002) 043503.

[3] R. Bernabei et al., Eur. Phys. J. C 18 (2000) 283.

[4] R. Bernabei et al., Eur. Phys. J. C 47 (2006) 263.

[5] R. Bernabei el al., La Rivista del Nuovo Cimento 26 (2003) 1.

[6] R. Bernabei et al., Phys. Lett. B 408 (1997) 439; P. Belli et al., Phys. Lett. B 460 (1999) 236; R. Bernabei et al., Phys. Rev. Lett. 83 (1999) 4918; P. Belli et al., Phys. Rev. C 60 (1999) 065501; R. Bernabei et al., Il Nuovo Cimento A 112 (1999) 1541; Phys. Lett. B 515 (2001) 6; F. Cappella et al., Eur. Phys. J.-direct C 14 (2002) 1; R. Bernabei et al., Eur. Phys. J. A 23 (2005) 7; Eur. Phys. J. A 24 (2005) 51; Astrop. Phys. 4 (1995) 45; in "The identification of Dark Matter", World Sc. Pub., Singapore, 1997, pp. 574.

[7] P. Belli et al., Astropart. Phys. 5 (1996) 217; Nuovo Cim. C 19 (1996) 537; Phys. Lett. B 387 (1996) 222; Phys. Lett. B 389 (1996) 783 err.; R. Bernabei et al., Phys. Lett. B 436 (1998) 379; P. Belli et al., Phys. Lett. B 465 (1999) 315; Phys. Rev. D 61 (2000) 117301; R. Bernabei et al., New J. of Phys. 2 (2000) 15.1; Phys. Lett. B 493 (2000) 12; Nucl. Instr. \& Meth A 482 (2002) 728; Eur. Phys. J. direct C 11 (2001) 1; Phys. Lett. B 527 (2002) 182; Phys. Lett. B 546 (2002) 23; in the volume "Beyond the Desert 2003", Springer, Berlin, 2003, pp. 365; Eur. Phys. J. A 27 s01 (2006) 35.

[8] R. Bernabei et al., Astropart. Phys. 7, 73 (1997); Nuovo Cim. A 110, 189 (1997); P. Belli et al., Astropart. Phys. 10, 115 (1999); Nucl. Phys. B 563, 97 (1999); R. Bernabei et al., Nucl. Phys. A 705, 29 (2002); P. Belli et al., Nucl. Instr. Meth. A 498, 352 (2003); R. Cerulli et al., Nucl. Instr. Meth. A 525, 535 (2004); R. Bernabei et al., Nucl. Instr. Meth. A 555, 270 (2005); Ukr. J. Phys. 51, 1037 (2006); P. Belli et al., Nucl. Phys. A 789, 15 (2007); Phys. Rev. C 76, 064603 (2007); Phys. 
Lett. B 658, 193 (2008); Eur. Phys. J. A 36, 167 (2008); Nucl. Phys. A 826, 256 (2009); Nucl. Instr. Meth. A 615, 301 (2010); Nucl. Instr. Meth. A 626-627, 31 (2011); J. Phys. G: Nucl. Part. Phys. 38, 015103 (2011); J. Phys. G: Nucl. Part. Phys. 38, 015107 (2011); Phys. Rev. C 85, 044610 (2012); A.S. Barabash et al., J. Instr. 6, P08011 (2011).

[9] P. Belli et al., Nucl. Instr. Meth. A 572, 734 (2007); Nucl. Phys. A 806, 388 (2008); Nucl. Phys. A 824, 101 (2009); Proceed. of the Int. Conf. NPAE 2008 (ed. INR-Kiev, Kiev), p. 473 (2009); Eur. Phys. J. A 42, 171 (2009); Nucl. Phys. A 846, 143 (2010); Nucl. Phys. A 859, 126 (2011); Phys. Rev. C 83, 034603 (2011); Eur. Phys. J. A 47, 91 (2011); Nucl. Instr. Meth. A 670 (2012) 10; Phys. Lett. B 711 (2012) 41; Nucl. Instr. Meth. A 704 (2013) 40; Eur. Phys. J. A 49 (2013) 24; Phys. Rev. C 87 (2013) 034607.

[10] R. Bernabei et al., Nucl. Instr. Meth. A 592, 297 (2008).

[11] R. Bernabei et al., Eur. Phys. J. C 56, 333 (2008).

[12] R. Bernabei et al., Eur. Phys. J. C 67, 39 (2010).

[13] R. Bernabei et al., Int. J. Mod. Phys. A 28, 1330022 (2013).

[14] R. Bernabei et al., Eur. Phys. J. C 62, 327-332 (2009).

[15] R. Bernabei et al., Eur. Phys. J. C 72, 1920 (2012).

[16] R. Bernabei et al., Eur. Phys. J. A 49, 64 (2013).

[17] R. Bernabei et al., Eur. Phys. J. C 72, 2064 (2012).

[18] R. Bernabei et al., J. of Inst. 7, P03009 (2012).

[19] D. Smith and N. Weiner, Phys. Rev. D 64 (2001) 043502; D. TuckerSmith and N. Weiner, Phys. Rev. D 72 (2005) 063509.

[20] K. Freese et al., Phys. Rev. D 71 (2005) 043516; Phys. Rev. Lett. 92 (2004) 111301.

[21] R. Bernabei et al., Int. J. Mod. Phys. D 13 (2004) 2127.

[22] F. S. Ling, P. Sikivie and S. Wick, Phys. Rev. D 70 (2004) 123503.

[23] J.D. Scargle, Ap.J. 263 (1982) 835; W.H. Press and G.B. Rybicki, Ap.J. 338 (1989) 277

[24] R. Bernabei et al., AIP Conf. Proceed. 1223 (2010) 50 arXiv:0912.0660. 
[25] R. Bernabei et al., J. Phys.: Conf. Ser. 203 (2010) 012040 arXiv:0912.4200; http://taup2009.lngs.infn.it/slides/jul3/nozzoli.pdf, talk given by F. Nozzoli.

[26] R. Bernabei et al., in the volume Frontier Objects in Astrophysics and Particle Physics, ed. S.I.F. (Vulcano, 2010), p. 157 arXiv:1007.0595.

[27] R. Bernabei et al., Can. J. Phys. 89 (2011) 11.

[28] R. Bernabei et al., Physics Procedia 37 (2012) 1095.

[29] R. Bernabei et al., arXiv:1210.6199.

[30] R. Bernabei et al., arXiv:1211.6346.

[31] P. Belli et al., Phys. Rev. D 84 (2011) 055014.

[32] A. Bottino et al., Phys. Rev. D 85 (2012) 095013.

[33] C.E. Aalseth et al., Phys. Rev. Lett. 106 (2011) 131301; C.E. Aalseth et al., Phys. Rev. Lett. 107 (2011)

[34] G. Angloher et al., Eur. Phys. J. C 72 (2012) 1971 (arXiv:1109.0702).

[35] R. Bernabei et al., arXiv:1308.5109. 